\title{
Inervación de los Músculos Fibulares Largo y Corto en Individuos Chilenos: Estudio Anatómico y Biométrico
}

\author{
Innervation of the Fibularis Longus and Brevis Muscles in Chilean Individuals: \\ Anatomic and Biometric Study
}

"Olave, E.; *** Galaz, C.; ${ }^{* * *}$ Retamal, P. \& **Cruzat, C.

OLAVE, E.; GALAZ, C.; RETAMAL, P. \& CRUZAT, C. Inervación de los músculos fibulares largo y corto en individuos chilenos: Estudio anatómico y biométrico. Int. J. Morphol., 28(4):1295-1300, 2010.

RESUMEN: La inervación de los músculos del compartimiento lateral de la pierna es conocida desde un punto de vista general y su dependencia del nervio fibular superficial (NFS) es descrita en la mayoría de los textos clásicos. Sin embargo, detalles del número de ramos nerviosos que reciben los músculos y a que nivel se encuentran sus puntos motores es poco conocido, sobretodo en nuestra población. Basado en lo anterior y con el propósito de aportar datos respecto a las variables mencionadas y determinar niveles de origen y localización de los puntos motores de los músculos fibulares largo y corto, se realizó un estudio anatómico y biométrico en 16 piernas formolizadas de individuos adultos, chilenos. Se realizó disección por planos, identificando en la parte proximal de la pierna el nervio fibular común (NFC) y su punto de división en nn. fibular superficial (NFS) y fibular profundo (NFP), localizando los ramos primarios (R1,R2,R3 o más) dirigidos a los músculos en estudio. Para determinar su punto de origen y punto de penetración en éstos, se identificó la parte más prominente del epicóndilo lateral del fémur, desde el cual se registró la distancia entre este punto de referencia y las variables mencionadas. Cuando hubo subdivisiones en ramos secundarios (RS), se registró también su punto de origen y punto de penetración en el vientre muscular. En el m. fibular largo se observó en los 16 casos R1 y R2; 13 casos con R1, R2 y R3; 5 casos con R1, R2, R3 y R4 y 1 presentó 5R. El R1 provino del NFC en 6 casos (37,5\%), del NFS en 9 (56,3\%) y del nervio cutáneo dorsal medial (división precoz de NFS) en 1 (6,3\%); el R2 se originó en el NFC en 2 casos (12,5\%), del NFS en 13 (81,3\%) y del nervio cutáneo dorsal medial en 1(6,3\%); el R3 fue emitido por el NFS en 11 casos y por el nervio cutáneo dorsal intermedio (división precoz NFS) en 2 casos.Los ramos primarios se subdividieron hasta en $5 \mathrm{RS}$. El m. fibular corto presentó 8 casos con R1; 5 con R1 y R2; 1 con R1, R2 y R3 y 2 con 4R. El R1 se observó en 11 casos (75\%) proviniendo desde el NFS y en 4 casos $(18,8 \%)$ provino del nervio fibular profundo accesorio; el R2 fue emitido por el NFS en 4 casos (50\%) y por el nervio fibular profundo accesorio en 4 (50\%); el R3 tuvo su origen en el nervio fibular profundo accesorio en 3 casos. Los ramos primarios de varias muestras se dividieron hasta en 5 RS. Los niveles donde los ramos nerviosos penetran en el vientre muscular son mostrados en tablas. Los hallazgos mostrados complementarán la anatomía de la inervación de los músculos del compartimiento lateral de la pierna.

PALABRAS CLAVE: Anatomía; Inervación; Puntos motores; Músculos fibulares largo y corto.

\section{INTRODUCCIÓN}

Los músculos fibulares largo y corto se encuentran localizados en el compartimiento lateral de la pierna y su inervación proviene del nervio fibular superficial, información que puede ser encontrada en diversos textos de Anatomía (Testut \& Latarjet, 1969; Rash \& Burke, 1985; Williams et al., 1995; Moore \& Dalley, 2002). Estos músculos tienen gran importancia en los movimientos de la articulación del tobillo, participando activamente en la extensión de la mis- ma y en la eversión del pie. Su función puede verse afectada por lesiones en el nervio fibular común (NFC) o superficial (NFS), ya que el primero se encuentra íntimamente relacionado con la articulación tibiofibular y con la cabeza y cuello de la fíbula, donde a este nivel el nervio es sensible a lesiones por traumatismos, por ejemplo, fracturas del cuello de la fíbula, contusiones de la cara lateral de la rodilla, luxaciones de la articulación tibiofibular, etc. (Sunderland, 1985).

\footnotetext{
* Facultad de Medicina, Universidad de La Frontera, Chile.

** Facultad de Medicina, Universidad Católica del Maule, Chile.

*** Alumnos Carrera de Medicina, Universidad Católica del Maule, Chile.
} 
Las descripciones encontradas muestran de forma general la inervación de estos músculos, sin embargo, conocimiento específico del número de ramos que los inervan es escaso, así como de los niveles de origen de cada ramo, así como de sus puntos de penetración en el vientre muscular.

Dado que la mayor parte de la información que tenemos sobre la anatomía proviene de estudios realizados en otras poblaciones, y considerando la importancia de conocer la inervación de los músculos fibulares en nuestro país, nos propusimos aportar datos biométricos del origen, longitud y puntos de penetración de los ramos destinados a ellos, sabiendo que pueden ser de utilidad en la estimulación eléctrica de sus puntos motores, así como soporte morfológico para estudios electromiográficos.

\section{MATERIAL Y MÉTODO}

Para el estudio se utilizaron 16 piernas, 9 izquierdas y 7 derechas, fijadas en formaldehído al 10\%, de individuos adultos, Chilenos, las cuales son parte del Laboratorio de Anatomía de la Facultad de Medicina de la Universidad Católica del Maule, Talca.

Inicialmente, se localizó la parte más prominente del epicóndilo lateral del fémur (EPCL), así como también la parte prominente del maléolo lateral (ML), registrando la distancia entre ambos puntos. El primero de ellos se utilizó como punto de referencia para medir el origen y los puntos de penetración de los ramos nerviosos. Se realizó disección por planos, alcanzando el nervio fibular común así como su punto de división en nervios fibular superficial y profundo. Se identificó él o los ramos dirigidos a los músculos fibular largo y corto, donde registramos con un caliper digital Mitutoyo, de precisión 0,05 mm, el nivel de origen del o los ramos principales (R1,R2, etc.), longitud y su división en ramos secundarios cuando los hubo (RS1,RS2, etc.).

De todas las muestras se realizaron esquemas y se fotografiaron las más representativas.

\section{RESULTADOS}

La distancia entre el EPCL y el ML fue en promedio de $373+19,0 \mathrm{~mm}$.

El nivel de división del NFC en nervios fibulares superficial y profundo se encontró en promedio a $65,2 \pm$ 7,27 mm del EPCL.

Respecto a la inervación del músculo fibular largo (mFL), se encontró que 2 ramos $(\mathrm{R} 1, \mathrm{R} 2)$ lo inervaban en 3 casos $(18,8 \%), 3$ ramos (R1,R2,R3) lo hacían en 6 casos $(37,5 \%), 4$ ramos se observaron en 6 casos $(37,5 \%)$ y 5 ramos en 1 caso $(6,2 \%)$.

En relación al nervio del cual se originó el R1, en 6 casos $(37,5 \%)$ provino del NFC (Fig.1), en 9 casos (56,3\%) lo hizo desde el NFS (Fig.2) y en 1 caso (6,2\%) se desprendió del nervio cutáneo dorsal medial (división precoz del NFS). El R2 provino del NFC en 1 caso $(6,2 \%)$, del NFS en 14 casos $(87,5 \%)$ y del nervio cutáneo dorsal medial en 1 caso $(6,2 \%)$. El R3 se originó desde el NFS en 11 casos $(84,6 \%)$ y del nervio cutáneo dorsal intermedio en 2 casos $(12,4 \%)$. El R4 provino en los 6 casos en que estuvo presente desde el NFS (100\%). Un caso que presentó un R5 fue emitido por el NFS.

El nivel de origen del R1 para el $\mathrm{mFL}$ fue en promedio de 67,8 \pm 12,2 mm del EPCL; el de R2 fue de 77,1 \pm 19,2 mm; el de R3 fue de 90,4 + 20,6 mm y el de R4 fue de 103,7 $\pm 32,6$ $\mathrm{mm}$.

Con respecto a la longitud de estos ramos nerviosos, el R1 tuvo en promedio $12,9 \pm 7,7 \mathrm{~mm}$; el R2 tuvo 22,9 $\pm 13,6$ $\mathrm{mm}$; el R3 tuvo $33,4 \pm 24,7 \mathrm{mmy}$ el R4 tuvo $18,6 \pm 7,8 \mathrm{~mm}$.

En relación a la división en RS (Fig. 3), de los 5 casos en que el R1 se dividió en RS, uno lo hizo en 2 RS, 3 lo hicieron en $3 \mathrm{RS}$ y uno en $5 \mathrm{RS}$; de los 6 casos en que R 2 se dividió, en 5 de ellos lo hizo en 2 RS y en uno en 3 RS; de los 6 casos en que R3 se dividió, en 4 de ellos lo hizo en 2 RS, en uno en 3 RS y en el otro en 4 RS. Esto permitió identificar los puntos motores, que en este músculo fluctuó entre 2 y 9 , con un promedio de 5 , donde el $50 \%$ de las muestras pre-

Tabla I. Promedios del nivel de penetración de los ramos nerviosos en el músculo fibular largo (mm).

\begin{tabular}{lrrrc}
\hline RAMOS & Directo & \multicolumn{1}{c}{ RS1 } & \multicolumn{1}{c}{ RS2 } & \multicolumn{1}{c}{ RS3 } \\
\hline R1 & $70,9+17,7$ & $77,7+9,1$ & $91,2+18,3$ & $103,2+20,3$ \\
R2 & $93,5+22,4$ & $94,9+33,8$ & $109,3+50,7$ & - \\
R3 & $117,6+22,9$ & $101,1+29,9$ & $118,3+29,2$ & $136,9+0,9$ \\
\hline
\end{tabular}




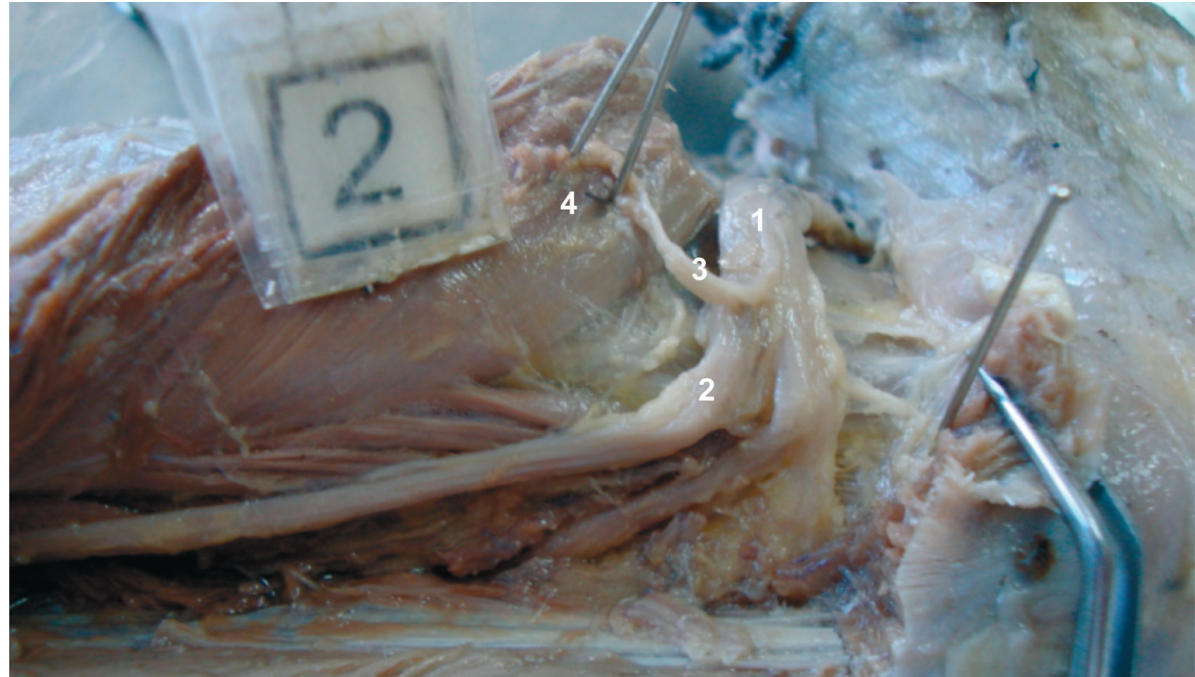

Fig. 1. Inervación del músculo fibular largo (4), 1. Nervio fibular común; 2. Nervio fibular superficial; 3. ramo para el músculo, con origen en el nervio fibular común.

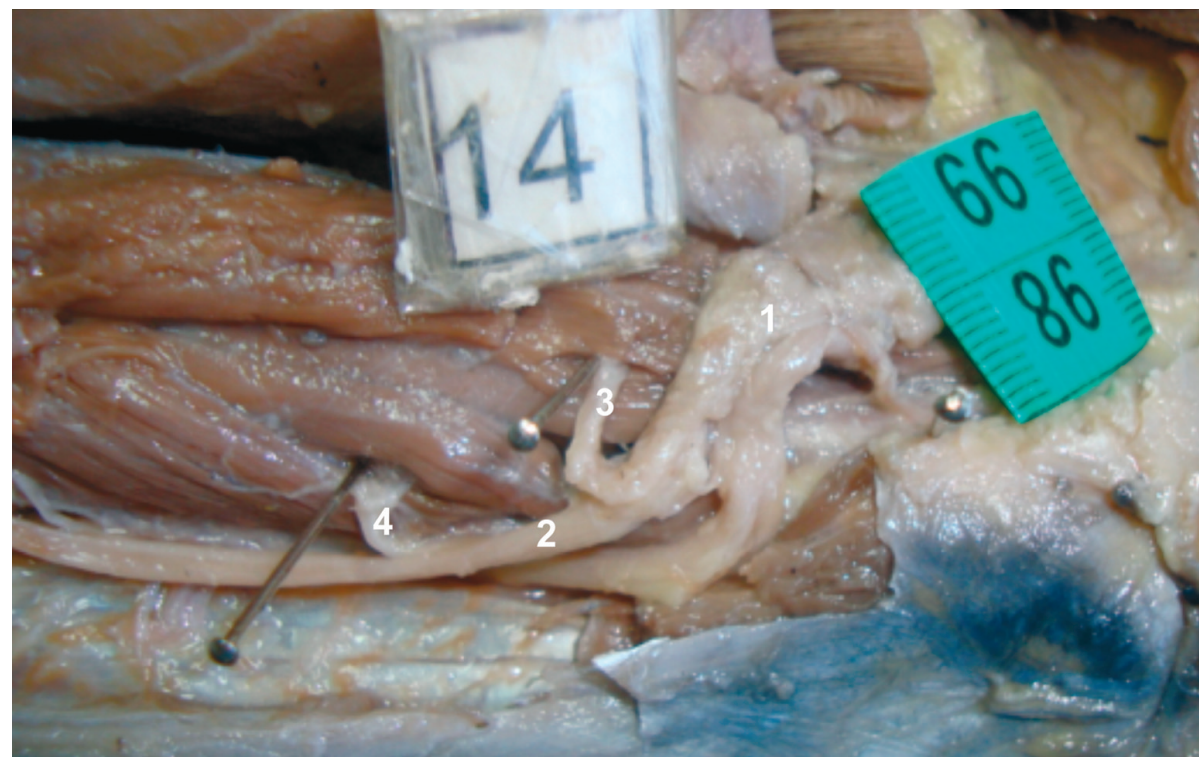

Fig.2. 1. Nervio fibular común; 2. nervio fibular superficial; 3 y 4. Ramos para el músculo fibular largo.

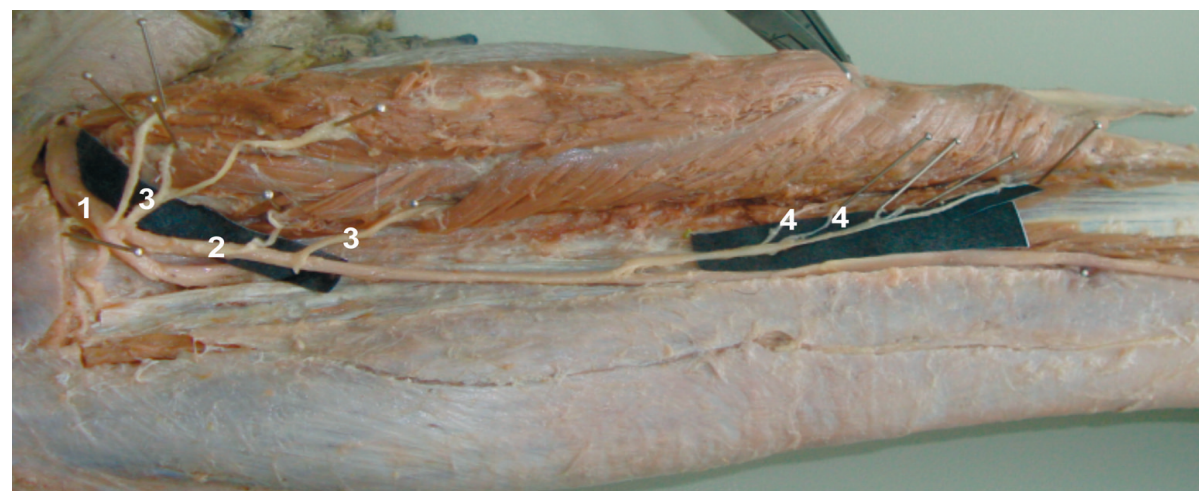

Fig.3. 1. Nervio fibular común; 2. nervio fibular superficial, 3. Ramos para el músculo fibular largo; 4. Ramos secundarios para el músculo fibular corto. sentó 4 y 5 puntos, donde el número menor ( 2 y 3 ) se visualizó en el $25 \%$ y el número mayor ( 8 y 9) en el $25 \%$ restante.

Los niveles del punto de penetración en el vientre muscular del mFL se muestran en la Tabla I.

Respecto a la inerva-ción del músculo fibular corto ( $\mathrm{mFC}$ ) (Fig. 4)), se encontró que 1 ramo (R1) lo inervaba en 8 casos (50\%), 2 ramos (R1, R2) lo hacían en 5 casos (31,3\%), 3 ramos se observaron en 1 caso $(6,2 \%)$ y 4 ramos en 2 casos $(12,5 \%)$.

En relación al nervio del cual se originó el R1, en 11 casos $(68,8 \%)$ provino del NFS, en 4 casos (25\%) lo hizo desde el NFP accesorio (Figs. 3 y 4 ) y en 1 caso $(6,2 \%)$ se desprendió del nervio cutáneo dorsal intermedio (división precoz del NFS). El R2 provino del NFS en 4 casos (50\%), del NFP accesorio en 4 casos $(50 \%)$. El R3 se originó desde el NFP accesorio en los 3 casos en que estuvo presente (100\%). El R4 provino en los 2 casos en el cual se observó desde el NFP accesorio.

El nivel de origen del R1 para el $\mathrm{mFC}$ fue en promedio de 147,2 + 32,5 mm del EPCL; el de $\mathrm{R} 2$ fue de 206,4 + 45,4 mm; el de R3 fue de $250,7 \mathrm{~mm}$.

Con respecto a la longitud de estos ramos nerviosos, el $\mathrm{R} 1$ tuvo en promedio $48,8 \pm 22,1$ $\mathrm{mm}$; el R2 tuvo $22,4 \pm 22,9 \mathrm{~mm}$.

En relación a la división de los ramos principales en RS, de los 8 casos en que R1 se dividió en RS, en 2 de ellos lo hizo en $2 \mathrm{RS}$, en 2 lo hizo en $3 \mathrm{RS}$, en 3 se dividió en $4 \mathrm{RS}$ y en uno en 


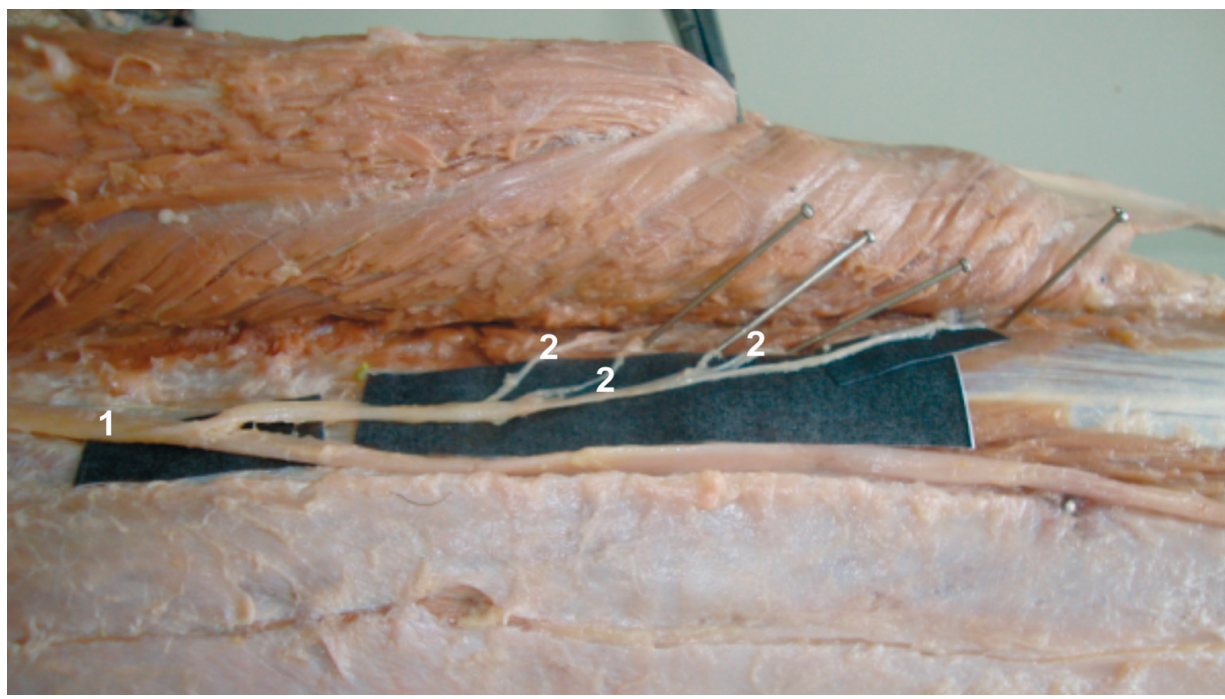

5 RS. El único caso en que R2 se dividió lo hizo en 2 RS. Los puntos motores en este músculo fluctuaron entre 1 y 5 , con un promedio de 3 , donde el $50 \%$ de las muestras presentó 2 y 3 puntos, donde el número mayor (4 y 5) se visualizó en el $37,5 \%$ y el número menor (1) en el $12,5 \%$ restante.

Los niveles del punto de penetración en el vientre muscular del $\mathrm{mFC}$ se muestran en la Tabla II.

Fig. 4. 1. Nervio fibular superficial; 2. Ramos para el músculo fibular corto.

Tabla II. Promedios del nivel de penetración de los ramos nerviosos en el músculo fibular.

\begin{tabular}{lccccc}
\hline Ramos & Directo & RS1 & RS2 & RS3 & RS4 \\
\hline R1 & $192,2+19,0$ & $177,4+23,4$ & $198,2+22,7$ & $199+17,6$ & $217,2+13,9$ \\
R2 & $225,6+41,2$ & - & - & - & - \\
R3 & $255,7+40,1$ & - & - & - & - \\
\hline
\end{tabular}

\section{DISCUSIÓN}

La inervación de los músculos de la pierna es conocida y descrita en los clásicos textos de Anatomía (Testut \& Latarjet; Williams et al.; Moore \& Dalley), sin embargo, conocimientos específicos de los niveles de origen de sus ramos y la localización de sus puntos motores es aún escasa, más aún en nuestra población.

El nervio fibular común se divide a nivel de la fosa poplítea en nervios fibular superficial y fibular profundo, donde el primero se dirige hacia el compartimiento lateral de la pierna y el segundo hacia el compartimiento anterior de ésta. En general, se describe que los músculos fibulares largo y corto reciben su inervación a a partir del NFS (Testut \& Latarjet; Rasch \& Burke; Dangelo \& Fattini, 1988; Williams et al.; Moore \& Agur, 2003).

Estudios realizados por Sunderland en 20 muestras han mostrado que el primer ramo para el músculo fibular largo puede provenir del NFC, el NFS o aún desde el NFP, datos con los cuales concordamos ya que en $37,5 \%$ de nues- tros casos este R1 provino del NFC y en 56,3\% desde el NFS. La descripción de Hollinshead (1976) señala que el músculo fibular largo es generalmente inervado por el NFS pero puede recibir también un ramo desde el NFC o del NFP. Escasa es la información sobre estos ramos musculares en individuos chilenos, a excepción del estudio de Méndez et al. (2009) quienes observaron que en todas las muestras de su serie (10) correspondientes a individuos chilenos el músculo fibular largo fue inervado por el NFS.

En relación el músculo fibular corto, en la mayor parte de nuestros casos su R1 provino del NFS y en una cuarta parte desde el nervio fibular profundo accesorio.

Con respecto al número de ramos para el músculo fibular largo, la mayor parte de las muestras estudiadas por Sunderland presentaron de 2 a 7 ramos y el músculo fibular corto presentó un ramo de inervación en casi todos los casos. En cambio, nuestro estudio mostró que el músculo fibular largo recibe 3 y 4 ramos principales en la mayor par- 
te de los casos $(75 \%)$, semejante al resultado de Méndez et $a l$. , quienes observaron 2 y 3 ramos principales para este músculo; el músculo fibular corto en nuestra serie recibió 1 y 2 ramos principales en la mayor parte de los casos $(81,3 \%)$, de los cuales el $50 \%$ presentó sólo 1.

El número de puntos motores encontrado fue variable, así en el mFL se observaron en promedio 5 puntos motores, encontrando en $50 \%$ de los casos 4 y 5 puntos; en el mFC observamos 3 en promedio, donde $50 \%$ de los casos tuvieron 2 y 3 puntos. Para el caso del mFL, nuestros resultados difieren de los encontrados por Méndez et al., quienes observaron entre 7 y 9 puntos motores.

Estos autores localizaron los puntos motores en relación a una línea trazada entre la cabeza de la fíbula y la porción distal del maléolo lateral y determinaron ramos musculares penetrando en las fibras superiores e inferiores, considerando en estas últimas una parte anterior y otra posterior. En nuestra serie, determinamos el nivel de ingreso de los ramos principales o sus ramos secundarios en el vientre muscular considerando como punto de referencia la parte más saliente del epicóndilo lateral del fémur, de tal modo de localizar con mayor posibilidad el sector donde se produce tal penetración. En el caso del $\mathrm{mFL}$, los datos de ambos estudios pueden complementarse, de tal modo que cuando sea necesario aplicar electrodos en los puntos descritos, la posición de ellos será más eficiente.

Con el propósito de definir aún más el sector en que estos músculos reciben su inervación y el origen de sus nervios, consideramos la distancia entre el EPCL y el ML, de tal modo que dividimos a ésta, en tercios y así podemos señalar para el caso del $\mathrm{mFL}$, que el nivel de origen de sus ramos se localizó en el tercio proximal de la distancia EPCL - ML y el nivel de localización de sus puntos motores corresponde principalmente al tercio proximal de la distancia EPCL-ML.

En relación al mFC, el nivel de origen de estos ramos se localizó principalmente en el tercio medio de la distancia EPCL-ML y el nivel de localización de los puntos motores se encuentra principalmente en el tercio medio de la distancia mencionada.

Desde el punto de vista de las distancias entre nuestro punto de referencia y el origen del R1, encontramos datos semejantes en descripción de Sunderland, en que señala que utilizando como referencia el epicóndilo femoral medial la menor distancia encontrada para el origen de este ramo para el $\mathrm{mFL}$ fue de $44 \mathrm{~mm}$ y la mayor de $91 \mathrm{~mm}$; para el $\mathrm{mFC}$ fue de $69 \mathrm{~mm}$ y $197 \mathrm{~mm}$, respectivamente. Nuestros resultados, utilizando el epicóndilo lateral fueron semejantes, para el mFL obtuvimos un registro de $56 \mathrm{~mm}$ para la distancia menor y de $92,7 \mathrm{~mm}$ para la mayor; en el caso del $\mathrm{mFC}$ fue de $86,9 \mathrm{~mm}$ y $185,1 \mathrm{~mm}$, respectivamente.

Sin duda que los datos obtenidos en esta investigación serán de interés para quienes deban electroestimular músculos atrofiados o para la realización de estudios electromiográficos, teniendo un buen soporte morfológico para localizar el origen y puntos motores de los ramos nerviosos dirigidos a los músculos del compartimiento lateral de la pierna de nuestra población, más aún por la escasez de literatura respecto a las variables mencionadas.

OLAVE, E.; GALAZ, C.; RETAMAL, P. \& CRUZAT, C. Innervation of the fibularis longus and brevis muscles in Chilean individuals: Anatomic and bimetric study. Int. J. Morphol., 28(4):1295-1300, 2010.

SUMMARY: The innervation of the muscles of the lateral compartment of the leg is known from a general point of view and its dependence of the superficial fibular nerve (SFN) is described in the majority of the classic texts. Nevertheless, there is little information regarding detail of the number of nervous branches that muscles receive, or the location of its motor points, principally in our population. The objective of this study was to contribute to anatomic knowledge of the mentioned variables and determine the origin levels of muscular branches and the motor points location in the fibularis longus and fibularis brevis muscles. We carried out an anatomical and biometric study in 16 cadaveric legs fixed in formaldehyde of adult, Chilean individuals. Dissections were made, identifying in the proximal part of the leg the common fibular nerve (CFN) and its division point in superficial (SFN) and deep (DFN) fibularis nerves, locating the primary branches (R1, R2, R3 or more) directed for the muscles in the study.To determine the origin level of muscular branches and motor point, we identified the most prominent part of the lateral epicondyle of femur, from which the distance was recorded between this point of reference and the mentioned variables. When there were subdivisions in secondary branches (SB), also we recorded its point of origin and motor point in the muscular belly. In the fibularis longus muscle we observed R1 and R2 in 16 cases; R1, R2 and R3 in 13 cases; R1, R2, R3 and R4 in 5 cases and $5 \mathrm{R}$ in one case. The R1 originated from the CFN in 6 cases $(37.5 \%)$, from the SFN in $9(56.3 \%)$ and from the medial dorsal cutaneous nerve (SFN precocious division) in 1 (6.3\%); The R2 originated in the CFN in 2 cases $(12.5 \%)$, of the SFN in $13(81.3 \%)$ and of the medial dorsal cutaneous nerve in $1(6.3 \%)$; the R3 was emitted by the SFN in 11 cases and by the intermediate dorsal cutaneous nerve (SFN precocious division) in 2 . The primary branches were subdivided even in $5 \mathrm{SB}$. The fibularis brevis muscle presented R1 in 8 cases; R1 and R2 in 5 cases; R1, R2 and R3 in one case and 4R in 2 cases. The R1 was observed in 11 cases ( $75 \%$ ) coming from the SFN and in 4 cases (18.8\%) originated from the accessory deep fibular nerve; the R2 was emitted by the SFN in 4 cases $(50 \%)$ and by the accessory deep fibular nerve in $4(50 \%)$; the R3 originated in the accessory deep fibular nerve in 3 cases. In various cases, the primary branches were divided even in 5 SB. The levels where the nervous branches penetrate in the muscular belly are shown in tables. These findings will complement the knowledge of the muscle innervation of the lateral compartment of the leg. 
OLAVE, E.; GALAZ, C.; RETAMAL, P. \& CRUZAT, C. Inervación de los músculos fibulares largo y corto en individuos chilenos: Estudio anatómico y biométrico. Int. J. Morphol., 28(4):1295-1300, 2010

\section{REFERENCIA BIBLIOGRAFICAS}

Hollinshead, H. Functional Anatomy of the Limbs and Back. Philadelphia, W.B. Saunders Company, 1976.

Méndez, G.; Coronado, R \& Suazo, I. Anatomical study of the fibularis longus muscle motor points and electrical stimulation therapy application. Int. J. Morphol., 27(3):699-703, 2009.

Moore, K. L. \& Agur, J. Fundamentos de Anatomía con Orientación Clínica. $2^{\mathrm{a}}$ Ed. Madrid, Panamericana, 2003.

Moore K. L.; Dalley AF. Anatomía con Orientación Clínica. $4^{\text {a }}$ Ed. Buenos Aires, Panamericana, 2004.

Rash, Ph. \& Burke, R. Kinesiología y Anatomía Aplicada. Buenos Aires, El Ateneo, 1985.

Sunderland, S. Nervios periféricos y sus lesiones. $2^{\mathrm{a}}$ Ed., Barcelona, Salvat, 1985.

Testut, L. \& Latarjet, A. Tratado de anatomía Humana. Barcelona, Salvat, 1969.

Williams P.L.; Warwick R.; Dyson M. \& Bannister L. H. Gray Anatomía. $37^{\text {th }}$ Ed. Rio de Janeiro, GuanabaraKoogan, 1995.

\author{
Dirección para correspondencia: \\ Prof. Dr. Enrique Olave Riffo \\ Facultad de Medicina \\ Universidad de La Frontera \\ Casilla 54-D \\ Temuco \\ CHILE
}

Email:eolave@ufro.cl

Recibido : 12-07-2010

Aceptado: 11-09-2010 